\title{
ANÁLISIS DEL DESGASTE DE LOS NEUMÁTICOS DE LOS TRACTOCAMIONES DE CARGA
}

\author{
Analysis of tiresusage of freight trucks
}

\section{EPISTEMUS}

ISSN: 2007-8196 (electrónico)

ISSN: 2007-4530 (impresa)

\section{Jezaharel Rocio Ibarra Salazar 1 Luis Felipe Romero Dessens 2 \\ Raquel Torres Peralta 3 \\ Federico Miguel Cirett Galan 4 José Luis Ochoa Hernández ${ }^{5}$}

Recibido: 14 de marzo de 2018, Aceptado: 30 de mayo de 2018

Autor de Correspondencia:

Ing. Jezaharel Rocio Ibarra Salazar

Correo: joylceo_dangel@hotmail.com

\section{Resumen}

En este documento se realiza un análisis que permita identificar las principales causas de desgaste asociados con los neumáticos de los tractocamiones de carga. Se realiza una evaluación sobre el desempeño y rentabilidad de neumáticos nuevos y reparados total o parcialmente. La obtención de estadísticas es una valiosa herramienta que apoya la toma de decisiones, permite realizar ajustes en los procesos para lograr incrementos en la productividad y rentabilidad de la empresa. Además, los beneficios de un mejor cuidado de los neumáticos no se limitan a lo económico, sino también a un impacto ambiental al reducir los desechos.

Palabras clave: Neumáticos, Tractocamiones de Carga, Causas de Desecho, Análisis Cuantitativo.

\section{Abstract}

In this paper an analysis is made to identify the main causes of freight trucks tires waste. An evaluation is made on the performance and profitability of new and repaired tires in whole or in part. The statistics analysis is a valuable tool that supports decision making, allows adjustments in processes to achieve increases in productivity and profitability of the company. In addition, the benefits of better tire care are not limited to economic, but also to an environmental impact by reducing waste.

Keywords: Tyres, Freight trucks, Waste causes, Quantitative analysis.

1 Universidad de Sonora, Departamento de Ingeniería Industrial / Correo:joylceo_dangel@hotmail.com

2 Universidad de Sonora, Departamento de Ingeniería Industrial / Correo: luisfelipe.romero@unison.mx

3 Universidad de Sonora, Departamento de Ingeniería Industrial / Correo: rtorres@industrial.uson.mx

4 Universidad de Sonora, Departamento de Ingeniería Industrial / Correo: fcirett@industrial.uson.mx

5 Universidad de Sonora, Departamento de Ingeniería Industrial / Correo: joseluis.ochoa@industrial.uson.mx 
Los datos analizados en este documento abarcan las operaciones de deshecho de neumáticos en la empresa de transporte del mes de agosto de 2015 a mayo de 2016. El análisis se centra en la Lista de Llantas de Desecho, donde se enlistan los siguientes datos:

- Consecutivo (Id de operación)

- Fecha en que se desecha el neumático

- Marca

- Diseño

- Medida

- El número de identificación del neumático (inicia con TP seguido de 5 dígitos, asegurando con ello que éste pertenece a la empresa).

- Número de serie

- Milímetros de piso al momento de desecharse, registrando el mínimo medido.

- Anotar O si el neumático desechado es original y $\mathrm{R}$ si era renovado.

- Finalmente, la clave de desecho. Número que identifica la causa del desecho, de 18 condiciones principales.

El análisis contempla la elaboración de tablas dinámicas para concentrar y agrupar la información, posteriormente construir series de tiempo, con valores totales, globales y porcentuales. Elaborar los gráficos pertinentes con el fin de realizar una interpretación sobre los resultados obtenidos y sustentar los hallazgos. A continuación, presentamos los resultados, exponiendo las principales causas de baja durante el período analizado.

\section{RESULTADOS}

Primeramente, se construyeron tablas dinámicas que muestran la frecuencia absoluta y relativa de cada condición (indicada con el número correspondiente 1-17) encontrada al momento de la baja, la cantidad de neumáticos originales y renovados, así como los milímetros de profundidad menores obtenidos de las tres lecturas efectuadas por el personal encargado de supervisión, monte y desmonte de neumáticos (una lectura de cada surco de la banda de rodado). Los resultados están categorizados por marca y expresado su participación porcentual por mes (figuras 4,5 y 6 ).

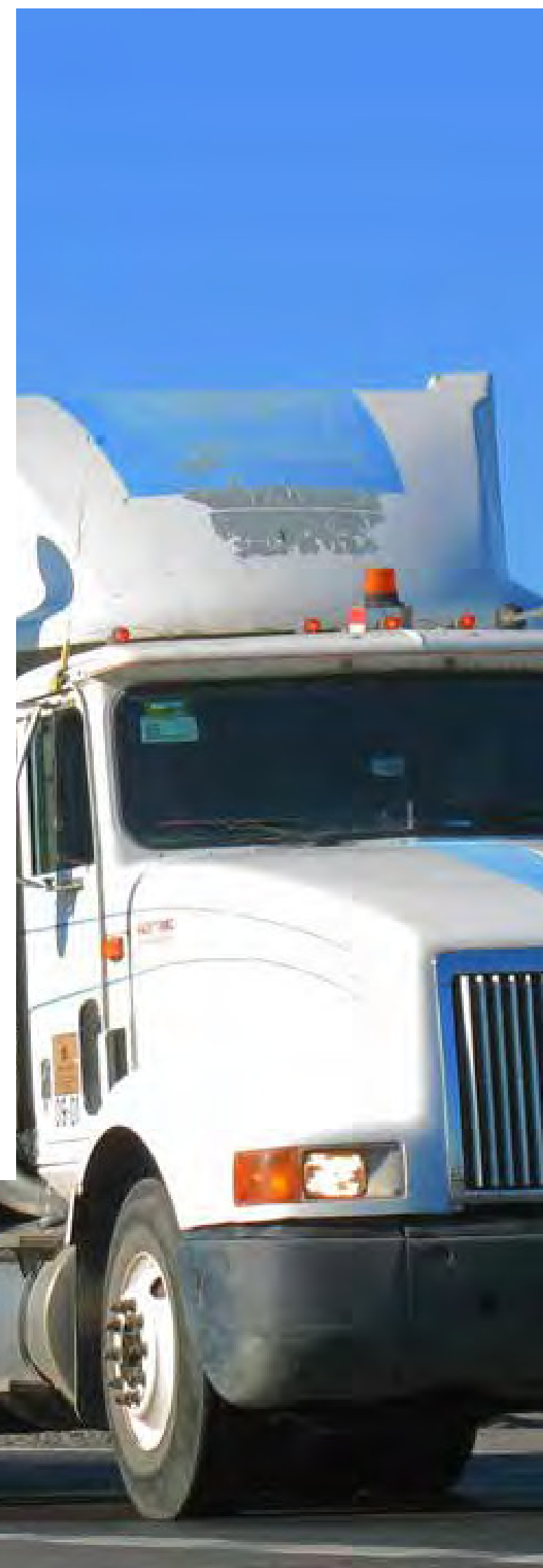

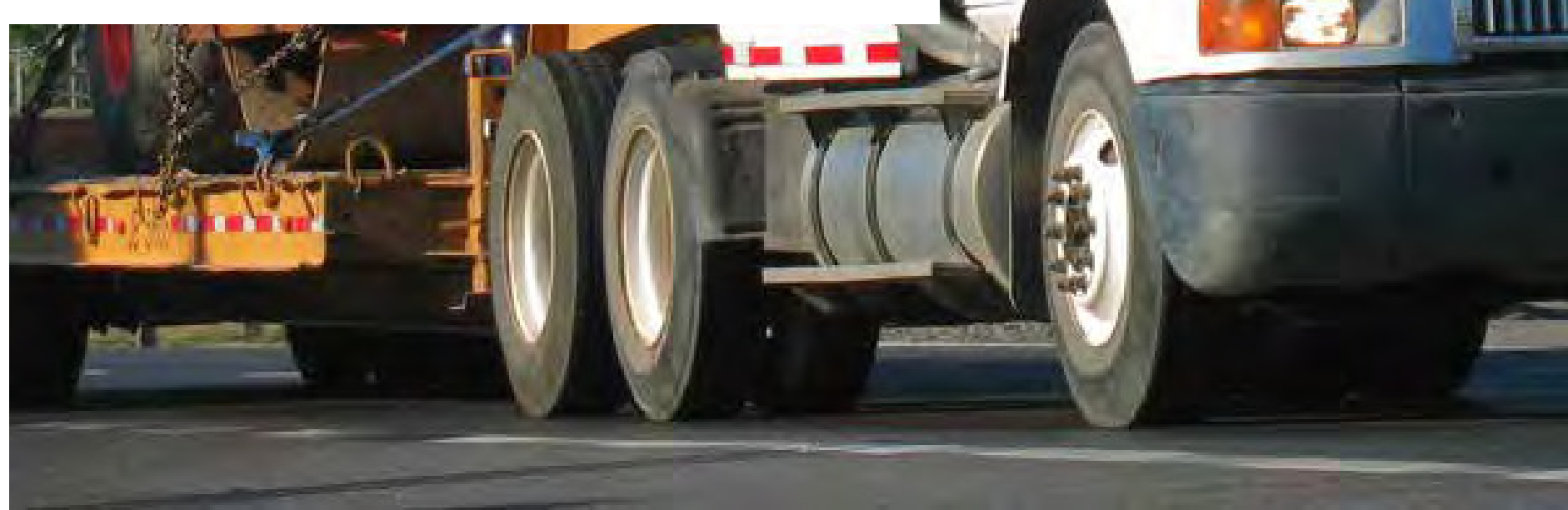




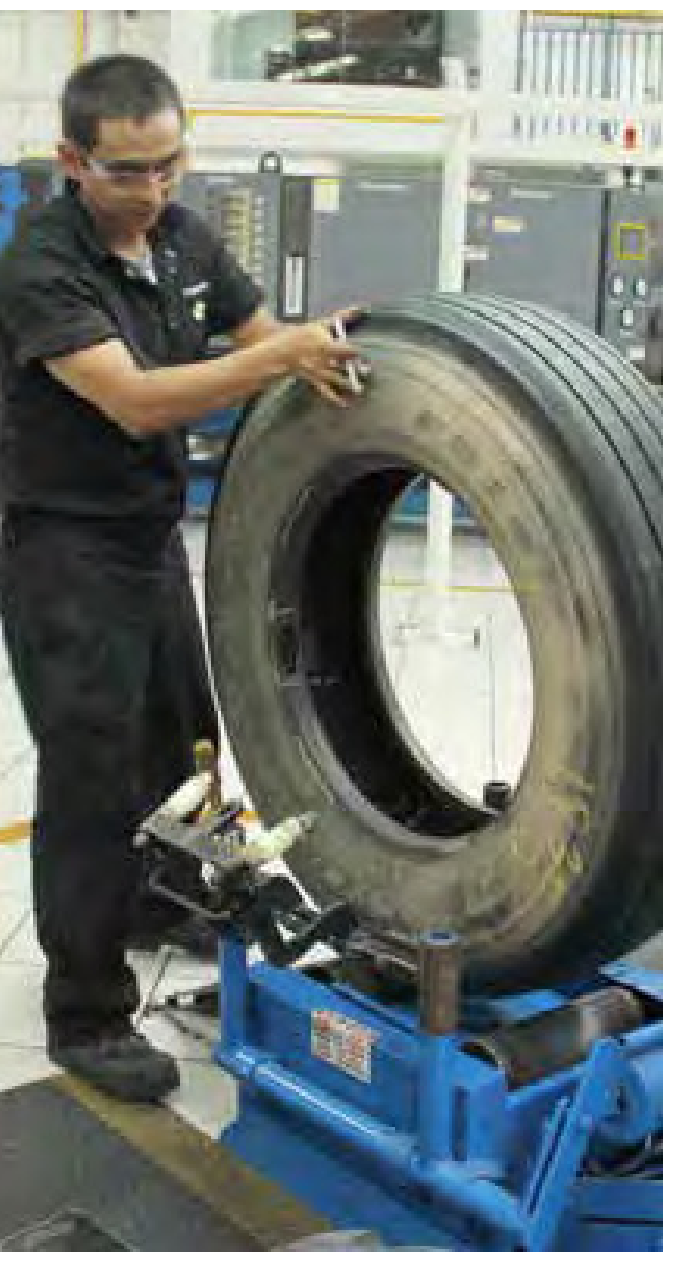

\begin{tabular}{|c|c|c|c|c|c|c|c|c|c|c|c|}
\hline \multirow{2}{*}{ MARCA } & \multicolumn{9}{|c|}{ CLAVE DE LA CONDICIÓN ENCONTRADA } & \multirow{2}{*}{$\begin{array}{c}\text { Total } \\
\text { general }\end{array}$} & \multirow{2}{*}{$\%$} \\
\hline & 2 & 3 & 4 & 5 & 8 & 10 & 14 & 16 & 17 & & \\
\hline AKUURET & & & 1 & & & & & & & 1 & $1 \%$ \\
\hline BF GOODRICH & & & & 1 & & 1 & & & & 2 & $2 \%$ \\
\hline BRIDGESTONE & 3 & 7 & 4 & 6 & 3 & 3 & 1 & 4 & 1 & 32 & $29 \%$ \\
\hline CONTINENTAL & 3 & 3 & & 4 & & & 2 & & & 12 & $11 \%$ \\
\hline DUNLOP & 3 & 1 & & 1 & & & & & 3 & 8 & $7 \%$ \\
\hline DYNATRAC & & & & 1 & & & & & & 1 & $1 \%$ \\
\hline FIRESTONE & 3 & 5 & 7 & 3 & 1 & 2 & 2 & 2 & 3 & 28 & $25 \%$ \\
\hline GENERAL & & & 1 & & & 1 & & & & 2 & $2 \%$ \\
\hline GOODYEAR & 1 & 2 & 1 & & & & & & 1 & 5 & $5 \%$ \\
\hline KUMHO & 1 & & & & & & & & & 1 & $1 \%$ \\
\hline MICHELIN & 4 & 1 & & 2 & 1 & & 1 & & 1 & 10 & $9 \%$ \\
\hline TECHK & & & & & 1 & & & & & 1 & $1 \%$ \\
\hline TOYO & 2 & & & & & & & & & 2 & $2 \%$ \\
\hline YOKOHAMA & 1 & 1 & 2 & 1 & 1 & & & & & 6 & $5 \%$ \\
\hline Total general & 21 & 20 & 16 & 19 & 7 & 7 & 6 & 6 & 9 & 111 & $100 \%$ \\
\hline$\%$ & $19 \%$ & $18 \%$ & $14 \%$ & $17 \%$ & $6 \%$ & $6 \%$ & $5 \%$ & $5 \%$ & $8 \%$ & $100 \%$ & \\
\hline
\end{tabular}

Figura 4. Concentrado de neumáticos por marca, según la clave de desecho para cada mes (datos: agosto 2015).

\begin{tabular}{|c|c|c|c|c|c|c|c|c|c|c|c|c|c|c|c|}
\hline \multirow{2}{*}{ MARCA } & \multicolumn{13}{|c|}{ MM ACTUAL } & \multirow{2}{*}{$\begin{array}{l}\text { Total } \\
\text { general }\end{array}$} & \multirow{2}{*}{$\%$} \\
\hline & 0 & 1 & 2 & 3 & 4 & 5 & 6 & 7 & 8 & 9 & 10 & 13 & 16 & & \\
\hline AKUURET & & & & & & & & & & 1 & & & & 1 & $1 \%$ \\
\hline BF GOODRICH & 1 & & 1 & & & & & & & & & & & 2 & $2 \%$ \\
\hline BRIDGESTONE & 9 & 1 & & 1 & 3 & 3 & 3 & & 6 & 2 & 2 & 1 & 1 & 32 & $29 \%$ \\
\hline CONTINENTAL & 1 & & & 1 & 1 & 3 & 2 & 2 & 1 & 1 & & & & 12 & $11 \%$ \\
\hline DUNLOP & 5 & & & & & & & 3 & & & & & & 8 & $7 \%$ \\
\hline DYNATRAC & & & & & 1 & & & & & & & & & 1 & $1 \%$ \\
\hline FIRESTONE & 7 & & & 4 & 1 & & 2 & 5 & 4 & 4 & 1 & & & 28 & $25 \%$ \\
\hline GENERAL & 1 & & & 1 & & & & & & & & & & 2 & $2 \%$ \\
\hline GOODYEAR & 1 & & & & & & & 2 & & 2 & & & & 5 & $5 \%$ \\
\hline КUМHO & 1 & & & & & & & & & & & & & 1 & $1 \%$ \\
\hline MICHELIN & 4 & & & & & & 2 & 1 & 1 & 1 & 1 & & & 10 & $9 \%$ \\
\hline TECHK & & & & & & & & & & 1 & & & & 1 & $1 \%$ \\
\hline TOYO & 1 & & & & & & & 1 & & & & & & 2 & $2 \%$ \\
\hline YОКОНАМА & 2 & & & & & 1 & & & 2 & & 1 & & & 6 & $5 \%$ \\
\hline Total general & 33 & 1 & 1 & 7 & 6 & 7 & 9 & 14 & 14 & 12 & 5 & 1 & 1 & 111 & $100 \%$ \\
\hline$\%$ & $30 \%$ & $1 \%$ & $1 \%$ & $6 \%$ & $5 \%$ & $6 \%$ & $8 \%$ & $13 \%$ & $13 \%$ & $11 \%$ & $5 \%$ & $1 \%$ & $1 \%$ & $100 \%$ & \\
\hline
\end{tabular}

Figura 5. Concentrado de neumáticos por marca, según los milímetros que tenía de remanente para cada mes. El menor número de milímetros es un indicador de mayor aprovechamiento o mejor desempeño (datos: agosto 2015). 


\begin{tabular}{|c|c|c|c|c|}
\hline \multirow{2}{*}{ MARCA } & \multicolumn{2}{|c|}{ ORIG/RENOV } & \multirow{2}{*}{ Total general } & \multirow{2}{*}{$\%$} \\
\hline & 0 & $\mathbf{R}$ & & \\
\hline AKUURET & & 1 & 1 & $1 \%$ \\
\hline BF GOODRICH & 1 & 1 & 2 & $2 \%$ \\
\hline BRIDGESTONE & 15 & 17 & 32 & $29 \%$ \\
\hline CONTINENTAL & 2 & 10 & 12 & $11 \%$ \\
\hline DUNLOP & 1 & 7 & 8 & $7 \%$ \\
\hline DYNATRAC & & 1 & 1 & $1 \%$ \\
\hline FIRESTONE & 8 & 20 & 28 & $25 \%$ \\
\hline GENERAL & & 2 & 2 & $2 \%$ \\
\hline GOODYEAR & & 5 & 5 & $5 \%$ \\
\hline KUMHO & & 1 & 1 & $1 \%$ \\
\hline MICHELIN & 1 & 9 & 10 & $9 \%$ \\
\hline TECHK & 1 & & 1 & $1 \%$ \\
\hline TOYO & & 2 & 2 & $2 \%$ \\
\hline YOKOHAMA & 1 & 5 & 6 & $5 \%$ \\
\hline Total general & 30 & 81 & 111 & $100 \%$ \\
\hline$\%$ & $27 \%$ & $73 \%$ & $100 \%$ & \\
\hline
\end{tabular}

Figura 6. Concentrado de neumáticos por marca, según su tipo: original o renovada, totales y porcentajes (Datos: agosto 2015).

Al construir una serie histórica de los totales mensuales se realizó un primer análisis de estos 10 meses, se pudo encontrar información relevante para apoyar la toma de decisiones. Primeramente, la cantidad de neumáticos desechados para este periodo fue de 1743 , de los cuales el $25 \%$ son originales, que al desecharse se pierde el casco y los milímetros de piso restantes, además de la oportunidad de renovarse (figura 7).

\section{Histórico Neumáticos $0 / R$}

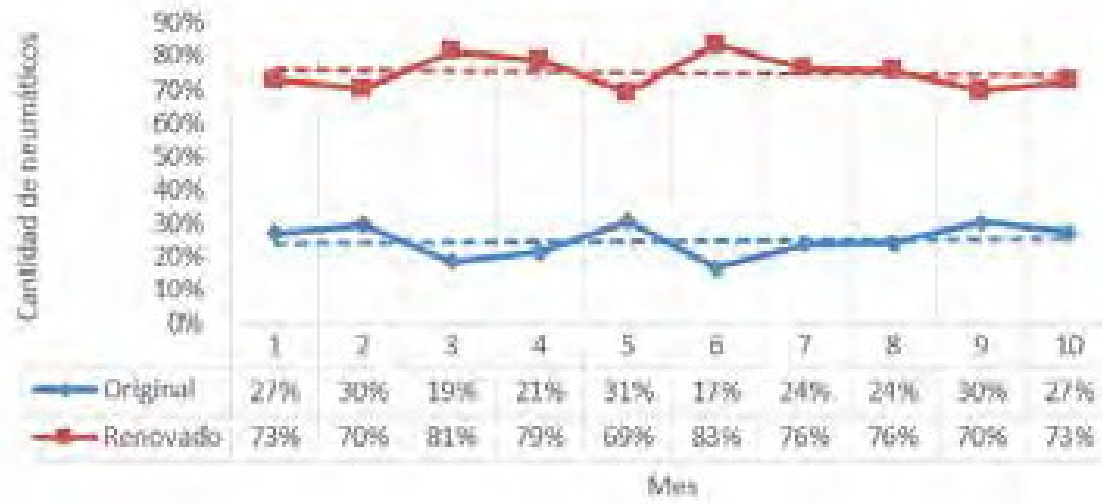

Figura 7. Neumáticos desechados por tipo.

Se promediaron los milímetros por neumáticos que se desechaban por mes, comparándolo con un valor promedio en general y para cada tipo, originales y renovados; de los $8014 \mathrm{~mm}$ de piso desechado, su promedio es de $4.6 \mathrm{~mm}$ de piso/neumático. De primera mano, uno esperaría encontrar la mayoría de los neumáticos desechados entre 0 y $3 \mathrm{~mm}$ de piso, según lo que establece una de las políticas de la empresa, pero los números demuestran que no se cumple debido a los pocos cuidados que se les brindan.

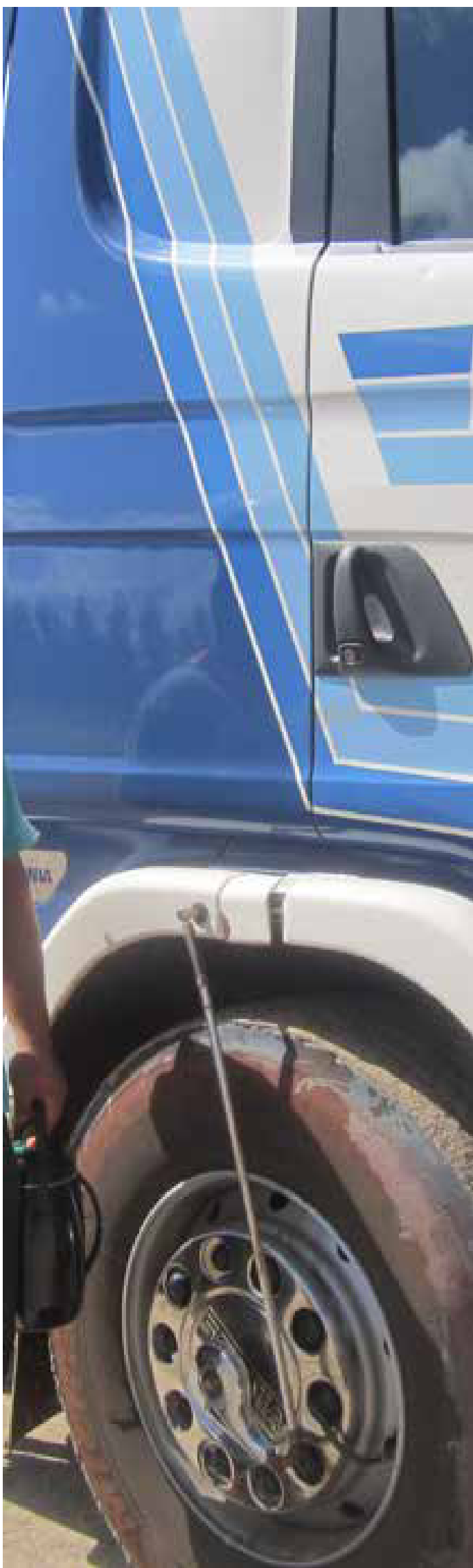




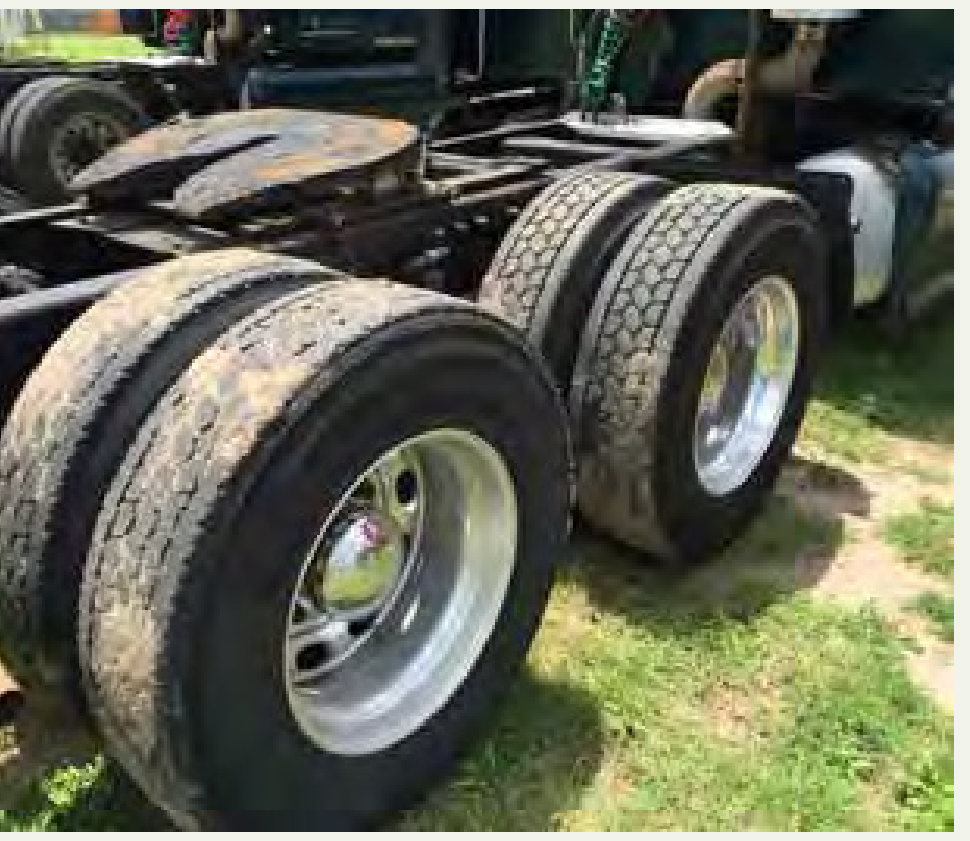

En los neumáticos originales, el promedio es de 3.9 $\mathrm{mm}$ /neumático lo que se registra; para los renovados se esperaría una disminución de dicho valor, pero por el contrario éstos se desechan a los $4.9 \mathrm{~mm} /$ neumático, al parecer no se está logrando aprovechar eficientemente el piso renovado (son $11.9 \mathrm{~mm}$ de piso en las renovaciones). Aunado a que en 6 de los 10 meses los valores promedio mensuales están por arriba de la media.

Respecto al mismo criterio, los valores que presentan mayor frecuencia al desecharse son 496 neumáticos con $0 \mathrm{~mm}$ (28\%), 201 con $8 \mathrm{~mm}$ (12\%), 168 de $7 \mathrm{~mm}(10 \%)$, 135 más con $5 \mathrm{~mm}(8 \%)$ y los 743 restantes con distintos milímetros (conformando el $42 \%$ restante), como se aprecia en la figura 8.

\section{Milimetraje de piso al desecharse}

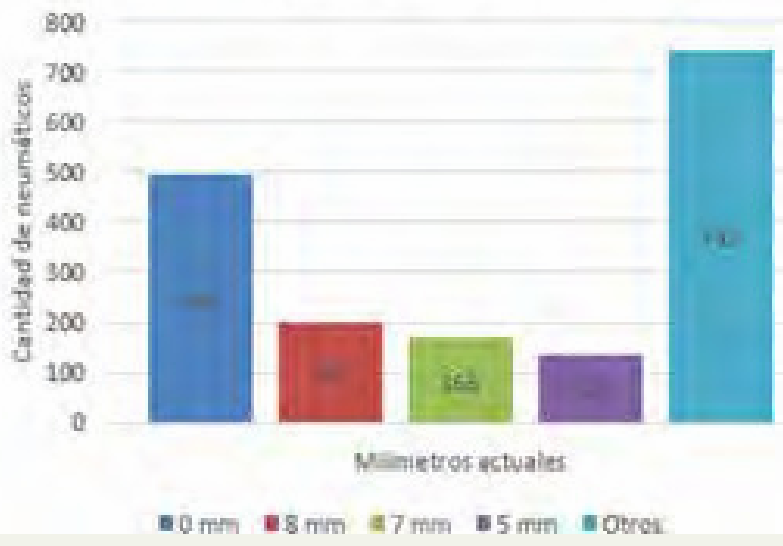

Figura 8. Milímetros de piso en los neumáticos al momento de darlos de baja.
Por otro lado, las causas de baja registradas por el responsable de la pila de desecho son:

1. Garantía aplicada

2. Arrancamiento banda de rodado

3. Daño en casco por impacto

4. Daño en costado

5. Daño en área de la ceja

6. Desgaste en línea central

7. Desgaste en hombros de la banda

8. Desgaste irregular provocado por problemas mecánicos

9. Desgaste por camber o alineación

10. Desgaste por debajo del TWI (permitido para renovación)

11. Desgaste por falta de rotación

12. Desgaste tipo sierra (convergencia o divergencia)

13. Falla en renovado

14. Fatiga del armazón (sobrecarga o rodado bajo)

15. Fuera de garantía

16. Objeto incrustado

17. Otro

18. Reparación mal hecha

De acuerdo a nuestro estudio, las 5 principales causas de desecho de neumáticos (78\% del total) son daños en los costados, arrancamientos de la banda de rodado, daño en el casco por impactos, daño en el área de la ceja y desgastes irregulares provocados por problemas mecánicos. La figura 9 muestra el porcentaje para cada una de ellas.

\section{Neumaticos desechados por Clave}

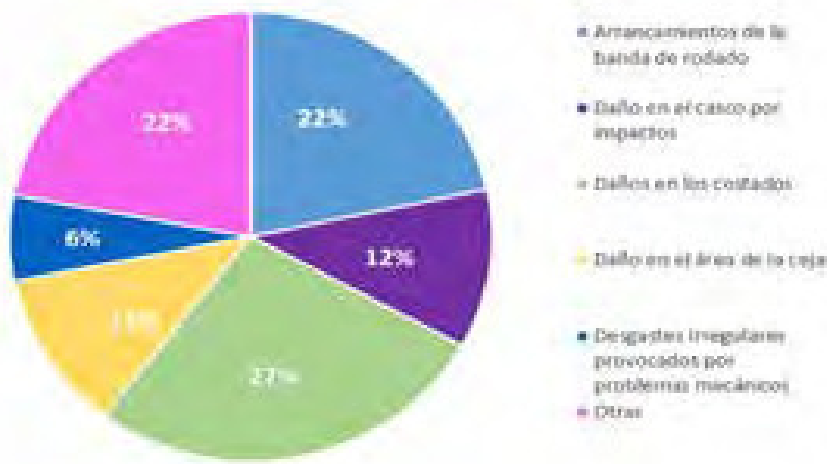

rıgura y. rorcentaje de neumaticos aesecnaaos, según la clave de condición encontrada al momento de darlos de baja. 
La primera de ellas que se distingue con la clave 4, daños en los costados, asociado a las maniobras del conductor, es decir, posiblemente el conductor no esté bien capacitado, no dimensione el vehículo, no presta atención al camino, entre otros.

El desprendimiento de la banda de rodeado es la segunda causa, quizá su origen se deba a altas temperaturas que ocasiona se desprenda la huella del neumático y se observa que se presenta más en los neumáticos renovados que en los originales que comúnmente encontramos estos pedazos sobre la cinta asfáltica.

Los cascos dañados por impacto y daños en el área de la ceja, se presentan en igualdad. Lo que comúnmente se denominan banquetazos son los del primer tipo; la ceja es la parte del neumático que entra en contacto con el rin, por lo que, si el neumático tiene una presión incorrecta tiende a dañarse en la ceja, esto también es atribuible al operador quien debe verificar las presiones en los neumáticos de la unidad antes de salir a trabajar.

Los desgastes irregulares en los neumáticos debido a diversos problemas mecánicos del vehículo, o clave 8, es la quinta causa con mayor aparición.

\section{DISCUSIÓN Y CONCLUSIONES}

De acuerdo a nuestro estudio, las principales causas de desecho de neumáticos se pueden atribuir a tres aspectos:

\section{Por la calidad del neumático y sus componentes.}

2.Por el tipo de camino, ruta y entorno donde transita.

3. Debido al operador (chofer) y su forma de conducir.

Las cuales tienen su origen en fallas mecánicas y en la operación de las unidades, pero pueden disminuirse significativamente tomando algunas medidas de control, por ejemplo: rediseñar el curso de capacitación y adiestramiento que se imparte a los operadores; establecer puntos de supervisión aleatorios y esporádicos para evaluar el mantenimiento que brinda el operador a la unidad asignada, entre otros.

Después de procesar la información se encontraron algunos puntos relevantes que no se habían contemplado en la captura de información de la pila de desecho, como el número de la unidad, la posición del neumático en el remolque o tractor, en caso de ser neumáticos originales, especificar si son del proveedor y si son renovados, identificar si pertenecieron a la empresa o fueron compradas al proveedor, por mencionar algunos.

El importante resaltar algunas limitantes por la falta de información capturada y digitalizada, además de que el procesamiento que se le dio a los datos no está completo, aún puede obtenerse más información relevante que permita contribuir de manera significativa en la toma de decisiones del área responsable, abriendo la puerta para futuras investigaciones o complementar la ya existente como puede ser los desprendimientos de las bandas de rodado en neumáticos renovados debido a posibles garantías con la empresa renovadora.

\section{BIBLIOGRAFÍA}

[1] Secretaría de Comunicaciones y Transportes, "Estadística Básica del Autotransporte Federal 2016", 2016.

[2] R. Hernández Sampieri, C. Fernández Collado, y P. Baptista Lucio, Metodología de la Investigación, Quinta. México, D.F.: McGraw-Hill, 2010.

[3] C. G. Sørensen y D. D. Bochtis, "Conceptual model of fleet management in agriculture", Biosyst. Eng., vol. 105, núm. 1, pp. 41-50, 2010.

[4] J. Pérez, S. Maldonado, y H. López-Ospina, "A fleet management model for the Santiago Fire Department", Fire Saf. J., vol. 82, núm. 5, pp. 1-11, 2016.

[5] H. Billhardt, A. Fernández, L. Lemus, M. Lujak, N. Osman, S. Ossowski, y C. Sierra, "Dynamic Coordination in Fleet Management Systems: Toward Smart Cyber Fleets", Intell. Syst. IEEE, vol. 29, núm. 3, pp. 70-76, 2014.

[6] Y. Hu, Y. Chiu, C. Hsu, y Y. Chang, "Identifying Key Factors for Introducing GPS-Based Fleet Management Systems to the Logistics Industry", Math. Probl. Eng., vol. 2015, núm. Article ID 413203, pp. 1-14, 2015.

[7] E. Demir, T. Bektaş, y G. Laporte, "A review of recent research on green road freight transportation", Eur. J. Oper. Res., vol. 237, núm. 3, pp. 775-793, 2014.

[8] H. R. Muñoz Chávez, "Impacto financiero de la división hombre-camión en una empresa de autotransporte de carga", Universidad Autónoma de Querétaro, 2008.

[9] Secretaría de Comunicaciones yTransportes, "Autotransporte Federal", 2016. .

[10]Michelin México, "Camión ¿Qué es una Ilanta?", Michelin Camión, 2016. [En línea]. Disponible en: http://michelin. softbox.mx/Camion/Camion/qllanta.asp. [Consultado: 29sep-2016].

[11]A. C. Reid, "Development and optimization of a wide base fea truck tire model for prediction of tire-road interactions", University of Ontario Institute of Technology, 2015.

[12]P. Barrezueta, "Diseño de un programa de mantenimiento y control operacional para neumáticos de camiones mezcladores de hormigón", Repos. Dsp., p. 7, 2012.

[13]Continental, "Buying tires", Technology, 2016. [En línea]. Disponible en: http://www.continental-tires.com/car/ technology/tire-knowledge/tirelexikon-2-0. [Consultado: 29-sep-2016].

[14]Coopertires de México, "Mantenimiento de la llanta", Seguridad, 2016. [En línea]. Disponible en: http://esmx. coopertire.com/Tire-Safety/Tire-Maintenance.aspx. [Consultado: 30-sep-2016].

[15]D. Andrade Mandon, "Implementación de un plan de mantenimiento correctivo y preventivo para la empresa Valorcon S.A. en el proyecto Vías de las Américas Sector 1 Campamento del Banco - Magdalena", Universidad Francisco de Paula Santander Ocaña, 2014.

[16]K. Wang, "Towards zero-defect manufacturing (ZDM)-a data mining approach", Adv. Manuf., vol. 1, núm. 1, pp. 6274, 2013. 